\title{
Thermal suppression of surface barrier in ultrasmall superconducting structures
}

\author{
W. V. Pogosov \\ Institute for Theoretical and Applied Electrodynamics, \\ Russian Academy of Sciences, Izhorskaya 13, 125412 Moscow, Russia
}

(Dated: January 19, 2021)

\begin{abstract}
In the recent experiment by Cren et al. [Phys. Rev. Lett. 102, 127005 (2009)], no hysteresis for vortex penetration and expulsion from the nano-island of $\mathrm{Pb}$ was observed. In the present paper, we argue that this effect can be associated with the thermoactivated surmounting of the surface barrier by a vortex. The typical entrance (exit) time is found analytically from the Fokker-Planck equation, written in the form suitable for the extreme vortex confinement. We show that this time is several orders of magnitude smaller than 1 second under the conditions of the experiment considered. Our results thus demonstrate a possibility for the thermal suppression of the surface barrier in nanosized low- $T_{c}$ superconductors. We also briefly discuss other recent experiments on vortices in related structures.

PACS numbers: 74.25.Bt, 74.25.Uv, 74.78.Na
\end{abstract}

\section{INTRODUCTION}

Vortex nucleation in an infinite type-II superconductor becomes energetically favorable at lower critical field $H_{c 1}$. It is however clear that real superconductors are always bounded, so that vortices can appear in a sample, at low temperatures, only by penetration through surfaces. This process was for the first time considered by Bean and Livingston ${ }^{1}$ within the framework of the London approximation. In their approach, single vortex was represented by a straight line entering a semi-infinite sample through its surface. Bean and Livingston have found a surface barrier preventing such an entry. The barrier disappears when $H$ is close to the thermodynamical critical field $H_{c}$, which is much larger than $H_{c 1}$ in superconductors with high values of the Ginzburg-Landau parameter $\kappa\left(H_{c} / H_{c 1} \sim \kappa / \ln \kappa\right)$. Physically, Bean-Livingston barrier is a consequence of the competition between the two factors: (i) the interaction between the vortex and the Meissner current, which tries to push the vortex line inside the sample; (ii) the Magnus attraction of a vortex to the surface, which can be understood in terms of the interaction between the vortex and its mirror image ("antivortex"). Similarly, surface barrier exists for the vortex exit from the sample. Bean-Livingston barrier plays an important role in the vortex dynamics near the superconductor surface and leads to pronounced hysteresis effects ${ }^{2}$.

As any potential barrier, Bean-Livingston barrier can be surmounted by thermal activation. This phenomenon was addressed by Petukhov and Chechetkin $\stackrel{3}{\text {, }}$ who have considered vortex nucleus as a half-loop spreading from the surface into the bulk. The profile of the surface barrier was calculated within the London approximation and the typical penetration time was estimated by using Fokker-Planck equation. The resulting formula is of Arrhenius type with the height of the surface barrier entering through the exponent. After substituting parameters typical for conventional low- $T_{c}$ materials, this height was estimated to be as large as $10^{3}-10^{5}$ in terms of $k_{B} T$, from which it was concluded that thermally activated vortex penetration is practically unobservable.

However, two decades later, after the discovery of high- $T_{c}$ materials, thermal activation over the surface barrier has attracted great attention. It was shown ${ }^{4}$ by Kopylov et al. that thermal surmounting of the barrier becomes possible in layered high- $T_{c}$ superconductors, where the mixed state structure is represented by quasi-2D pancake vortices. Burlachkov $\sqrt{5}$ has analyzed the same phenomenon for high- $T_{c}$ superconductors of $\mathrm{YBaCuO}$ type with $3 \mathrm{D}$ vortex lines. He has demonstrated that the height of the surface barrier in these compounds is dramatically suppressed, compared to low- $T_{c}$ materials, to $10-100 k_{B} T$, which leads to experimentally observable rates for thermally activated vortex penetration and expulsion. Indeed, these conclusions agree with numerous experiments, see e.g. Refer as well as the theoretical paper ${ }^{\mathbb{7}}$.

Thus, it is generally believed that thermally activated flux penetration and expulsion in low- $T_{c}$ superconductors is not possible, while for the case of high- $T_{c}$ superconductors it is quite standard. However, in the very recent experiment $t^{[8}$ by Cren et al. no hysteresis for vortex entry and exit was found in a superconducting nano-island of $\mathrm{Pb}$ having an extremely small thickness $(\approx 5.5 \mathrm{~nm})$. Lateral dimension of this sample was so small $(\sim 100 \mathrm{~nm})$ that it could accommodate only one vortex before turning to the normal state. This regime was referred in Ref $[8]$ as ultimate vortex confinement.

The aim of the present paper is to show that thermal fluctuations can be responsible for the effective suppression of the surface barrier observed in the experiment of Crer ${ }^{8}$ et al. . This cannot be firmly proved by using previously developed approaches to the thermal activation over the surface barrier, since all these schemes are based on the London theory, while this theory is not applicable for small-sized samples with lateral dimensions of the order of the 
vortex core size. Moreover, under these conditions, vortex position is not an appropriate variable, because an initial stage of vortex nucleation is associated with a "nascent" vortex, considered for the first time by Kramer 10 . "Nascent" vortex represents an area at the sample's edge, where the order parameter is suppressed, but it does not vanish yet. As we show, the height of the potential barrier, under the experimental conditions of Ref ${ }^{8}$, is mostly due to the vortex core nucleation rather than because of the motion of already formed vortex towards the island center. Thus, the origin of the surface barrier in the limit of a very small sample size is not identical to the one for the traditional Bean-Livingston barrier. This feature has already been discussed in Ref $\underline{9}$.

In the present paper, in order to circumvent these difficulties, we use the following alternative scheme. (i) We first solve Ginzburg-Landau equations approximately by using lowest Landau level approximation for the order parameter and determine the surface barrier in terms of the populations of corresponding levels. The island we consider is so small that it can accommodate only one vortex. Hence, to describe a barrier with a reasonable accuracy, it is sufficient to take into account only two levels (with winding numbers 0 and 1), which correspond to the vortex-free and one-vortex states. Populations of these levels are then used as "good" variables instead of the vortex position. Notice that there are no essential difficulties in extending the number of variables by taking into account more levels, which can be necessary when considering larger structures. (ii) We then estimate "viscosity" coefficients associated with the motion of order parameter projected on the two Landau levels. Demagnetization effects are included into the consideration. (iii) At the final step, we solve the backward Fokker-Planck equation, again in terms of the Landau levels populations. Our main result is an analytical expression for the typical time for thermally activated vortex entry (exit). Notice that the lowest Landau level representation for the order parameter in mesoscopic superconductors was proposed in Refs. 11 and then used very widely.

Our calculations show that the height of the surface barrier, under the conditions of the experiment considered, is approximately $20 k_{B} T$ that is more typical for high- $T_{c}$ materials than for conventional low- $T_{c}$ superconductors. The major reasons for the potential barrier to be not so high are (i) very small island thickness, (ii) very small island lateral dimensions which result in the substantial suppression of the order parameter, (iii) relatively high sample's temperature $^{8}(\approx 4.3 \mathrm{~K})$. The preexponential "attempt time" is quite small - mainly because of the small system sizes, which result in a weak magnetic response of the island. This time was estimated to be of the order of $10^{-15}-10^{-14} \mathrm{~s}$ which is much smaller than the similar quantity ${ }^{4}$ for high- $T_{c}$ layered superconductors with pancake vortices $\left(\sim 10^{-12}\right.$ $\mathrm{s})$. The resulting first passage time $\tau$ both for the vortex entry and exit is also quite small, $\tau \sim 10^{-5}-10^{-4} \mathrm{~s}$.

Thus, the results we here present demonstrate that the thermally-assisted suppression of the surface barrier can occur in nanosized samples made of low- $T_{c}$ superconductors, which are already available for experimental investigations.

Since nanosized superconductors might be perspective for technological application (see e. g. Ref $14 \mid 15$ ) and are even already used (for instance, for single-photon detection purposes ${ }^{16}$ ), the control of fluctuations in such structures is quite important.

This paper is organized as follows. In Section II, we study surface barrier within the Ginzburg-Landau theory. In Section III we estimate viscosity coefficients, with taking into account demagnetization effects. In Section IV, we solve the backward Fokker-Planck equation and determine first passage times both for the vortex penetration and expulsion. We conclude in Section V.

\section{SURFACE BARRIER}

In this section, we determine approximately the height of the potential barrier for vortex exit and entry under the conditions of experiment ${ }^{8}$. Our results are applicable as long as the sample's radius remains comparable to the coherence length $\xi(T)$.

\section{A. Main parameters}

The superconducting island studied in Ref $[8$ had a "smoothed" hexagonal shape with a small thinner region in its center, as seen from Fig. 1(b) of Ref. 8 . The smallest lateral size of the sample was 110 nm, while the thickness was $d=5.5 \mathrm{~nm}$. Sample temperature was $T=4.3 \mathrm{~K}$. The transition between the vortex-free and one-vortex states, as obtained by STS measurements, occurred at the applied field of $0.235 \mathrm{~T}$. The transition was reversible within $3 \%$. The reversibility implies that at this field energies of vortex-free and one-vortex phases become equal.

There was a disordered layer between the island and the Si substrate, which limitates ${ }^{8}$ the quasiparticle mean free part to $l \approx 2 d$. The zero-temperature coherence length was estimated ${ }^{8}$ by using an expression for dirty superconductors $\approx \sqrt{\xi_{0} l}$, where $\xi_{0}=80 \mathrm{~nm}$ is the coherence length in bulk $\mathrm{Pb}$. This yields $\approx 30 \mathrm{~nm}$. The coherence length $\xi(T=4.3 K)$ should be larger, but it is not easy to find its precise value theoretically because of the small system sizes (and nontrivial shape), which makes the microscopic physics quite complex 17 . However, it was possible to extract the coherence length 
directly from the measured zero-bias conductance profile in the vortex core, which yielded the value of $40-45 \mathrm{~nm} 18$. In this paper, we assume a value of $48 \mathrm{~nm}$. For the penetration depth, we use the usual expression 17 for dirty superconductors, $\lambda(T) \simeq 0.615 \lambda_{0} \sqrt{\frac{\xi_{0} / l}{\left(1-T / T_{c}\right)}}$ where $\lambda_{0} \simeq 40 \mathrm{~nm}$ is the penetration depth in bulk $\mathrm{Pb}, T_{c}=7.2 \mathrm{~K}$. This gives $\lambda(4.3 K) \simeq 102 \mathrm{~nm}$. Notice that the use of the prefactor 0.615 is, in fact, rather relative. The Ginzburg-Landau parameter, corresponding to the above numbers, is $\kappa \approx 2$. When considering the diamagnetic response of the island, one has to keep in mind that effective $\kappa$ is much higher $\underline{19}$, since $d \ll \lambda(T)$.

Although the island has a rounded hexagonal shape, it is actually quite similar to a disc (see Fig. 1(b) of Ref. ${ }^{8}$ ). Therefore, we model it by the disc. As for its diameter, we use the value of $150 \mathrm{~nm}$, which corresponds approximately to the largest ("bottom") lateral dimension of the island. This value together with 48 nm for the coherence length will allow us to reproduce the experimentally found field for the transition between the vortex-free and single-vortex states $(0.235 \mathrm{~T})$.

Note that the radius of the disc in terms of the coherence length is approximately 1.55. This regime indeed corresponds to the "ultimate vortex confinement": the disc is able to accommodate only one vortex before the transition to the normal state, see e.g. Ref. 20 .

\section{B. Basic estimates}

Let us make some basic estimates. We compare the thermal energy $k_{B} T$ with the energy required to create a vortex nucleus, i.e., to suppress the order parameter to zero within the volume $\sim \xi(T)^{2} d$. The similar idea is applied when calculating the Ginzburg-Levanyuk number ${ }^{21}$. Within the Ginzburg-Landau theory, the energy gain due to the vortex nucleus is

$$
\sim \frac{H_{c}(T)^{2}}{2 \mu_{0}} \xi(T)^{2} d
$$

where $H_{c}(T)$ is the thermodynamical critical field, given by

$$
H_{c}(T)=\frac{\Phi_{0}}{2 \pi \sqrt{2} \xi(T) \lambda(T)} .
$$

For the experiment performed at $T=4.3 \mathrm{~K}$ we obtain the following estimate for the ratio:

$$
k_{B} T\left(\frac{H_{c}(T)^{2}}{2 \mu_{0}} \xi(T)^{2} d\right)^{-1} \sim 10^{-2}
$$

which is basically not so small. Notice that the order parameter is strongly suppressed in the vicinity of the transition between vortex-free and one-vortex states. This should reduce condensation energy and enhance the ratio (3) in several times. Also, we expect that the barrier height to be much lower than the above estimate due to an additional reason: vortex nucleation is associated mainly with the redistribution of the density of superconducting electrons inside the island rather than with the change of their total number. Thus, the ratio of $k_{B} T$ and the barrier height has to be of the same order as in high- $T_{c}$ superconductors 5 , where rates for thermally activated flux penetration and expulsion are observable. Below we show that this is indeed the case.

\section{Calculation of the surface barrier}

We start with the dimensionless Ginzburg-Landau functional for the energy of the disc in the superconducting phase compared to the normal state:

$$
F=\frac{H_{c}(T)^{2}}{\mu_{0}} \xi(T)^{2} d \int_{0}^{2 \pi} d \varphi \int_{0}^{r_{0}} r d r\left(-|f|^{2}+\frac{1}{2}|f|^{4}+|(-i \nabla-\mathbf{a}) f|^{2}\right)
$$

where integration is performed over the disc cross section (cylindrical coordinate system is used), $r_{0}=R / \xi(T)$ is the dimensionless radius of the disc, $f$ is the dimensionless order parameter, $a=h_{e} r / 2$ is the vector potential, which has only an azimuthal component, $h_{e}=H / H_{c 2}(T)$ is the external magnetic field measured in terms of the upper critical field $H_{c 2}(T)=\Phi_{0} / 2 \pi \xi(T)^{2}$, and all the distances are measured in units of $\xi(T)$. Due to small disc sizes, the external magnetic field penetrates the sample almost totally, so that the magnetic field inside the disc is essentially equal to 
the external field. Since the disc thickness $d$ is much smaller than $\xi(T=4.3 K)$, the order parameter does not vary in $z$ direction. Hence, the problem both for the order parameter and barrier height is effectively two-dimensional.

The order parameter can be represented as a Furrier expansion

$$
f(r, \varphi)=\sum_{n} c_{n} \varphi_{n}(r) e^{-n \varphi}
$$

In the vortex-free state, only one coefficient among $c_{n}$ 's is nonzero, namely $c_{0}$. In the one-vortex state, a nonzero coefficient is $c_{1}$. The disc we consider is so small (in terms of $\xi(4.3 K)$ ) that it can accommodate only one vortex. Therefore, with good accuracy, we can map the order parameter on the subspace with only two nonzero components:

$$
f(r, \varphi) \simeq c_{0} \varphi_{0}(r)+c_{1} \varphi_{1}(r) e^{-i \varphi}
$$

The superposition (6) actually describes vortex exit or entrance. If we start with the vortex-free state $\left(c_{1}=0\right)$, an increase of $c_{1}$ first leads to the suppression of the order parameter at some spot on the disc edge. This suppression can be interpreted as a formation of a vortex nucleus or a "nascent" vortex 10 . At certain value of $c_{1}$, the order parameter in the center of this nucleus gets suppressed to zero, and the phase of the order parameter now changes by $2 \pi$ when turning around this topological defect. Thus, the real vortex is formed. Further increasing of $c_{1}$ leads to the vortex displacement towards the disc center, with its position $r_{v}$ given simply by the condition: $\left|c_{0}\right| \varphi_{0}\left(r_{v}\right)-\left|c_{1}\right| \varphi_{1}\left(r_{v}\right)$, which follows directly from Eq. (6). If we now start with the one-vortex state $\left(c_{0}=0\right)$, an increase of $c_{0}$ results in the vortex displacement towards the disc edge until it leaves the island.

Further simplifications come by using, for $\varphi_{0}(r)$ and $\varphi_{1}(r)$, the eigen functions for the kinetic energy operator corresponding to winding numbers $L=0$ and $L=1$, respectively, and to zero radial quantum number (nodeless wave functions). The latter corresponds to the lowest eigen value for the kinetic energy operator, see e.g. Ref.22. This choise for $\varphi_{0}(r)$ and $\varphi_{1}(r)$ can be justified by noting that at the transition to the normal state, the first Ginzburg-Landau equation can be linearized, i.e., reduced to the eigen value equation for the kinetic energy operator (with the eigen value 1). Therefore, in the vicinity of the transition, $\varphi_{n}(r)$ can be closely approximated by the the eigen functions for the same operator. This regime is indeed realized in the superconducting disc under conditions of "ultimate vortex confinement", where the transition between the one-vortex and vortex-free states occurs at high fields, $H \approx H_{c 2}(T)$, so that the density of superconducting electrons is strongly suppressed. In cylindrical coordinates, eigen value equation reads

$$
-\frac{1}{r} \frac{d}{d r}\left(r \frac{d \varphi_{L}}{d r}\right)+\varphi_{L}\left(\frac{h_{e} r}{2}-\frac{L}{r}\right)^{2}=\varepsilon_{L} \varphi_{L}
$$

This equation can be solved analytically:

$$
\varphi_{L}=r^{L} \varepsilon_{L}^{L / 2} \exp \left(-\frac{h_{e} r^{2}}{4}\right) \Phi\left(\frac{h_{e}-\varepsilon_{L}}{2 h_{e}}, L+1, \frac{h_{e} r^{2}}{2}\right),
$$

where $\Phi$ is Kummer function. Eq. (7) has to be supplemented by the boundary condition at the superconductor/vacuum interface

$$
\left.\frac{d \varphi_{L}}{d r}\right|_{r=r_{0}}=0
$$

Values of $\varepsilon_{L}$ for given $h_{e}, r_{0}$, and $L$ can be found numerically from Eqs. (8) and (9).

We now treat $c_{0}$ and $c_{1}$ as variational parameters and calculate disc energy, as given by Eq. (4), as a function of these two parameters. Thus the energy of the system is obtained as a biquadratic function of variational parameters $c_{0}$ and $c_{1}$. The resulting expression can be minimized with respect to $c_{0}$ and $c_{1}$. Without loss of generality, $c_{0}$ and $c_{1}$ can be taken real and positive. Although this method is not exact, we expect that it allows one to obtain rather accurate estimate of the potential barrier height for such a small island with the radius only slightly exceeding the vortex core size 23 .

The energy of the island, obtained from Eqs. (4)-(9), is given by

$$
F=\frac{H_{c}(T)^{2}}{\mu_{0}} \xi(T)^{2} d\left(c_{0}^{2} \alpha_{0}+c_{1}^{2} \alpha_{1}+c_{0}^{4} \beta_{0}+c_{1}^{4} \beta_{1}+2 \gamma c_{0}^{2} c_{1}^{2}\right),
$$


where $(L=0,1)$

$$
\begin{aligned}
\alpha_{L} & =\left(\varepsilon_{L}-1\right) \int_{0}^{r_{0}} \varphi_{L}^{2} r d r \\
\beta_{L} & =\frac{1}{2} \int_{0}^{r_{0}} \varphi_{L}^{4} r d r \\
\gamma & =\int_{0}^{r_{0}} \varphi_{0}^{2} \varphi_{1}^{2} r d r .
\end{aligned}
$$

In the range of parameters corresponding to the ultimate vortex confinement, $\alpha_{L}, \beta_{L}, \gamma \sim 0.1-0.5$. Let us stress that these numbers are internally dependent on the external field.

The energy of the island in the vortex-free state $F(L=0)$ can be found from Eq. (10) by minimization of $F$ with respect to $c_{0}$, while keeping $c_{1}=0$ :

$$
F(L=0)=-\frac{H_{c}(T)^{2}}{\mu_{0}} \xi(T)^{2} d\left(\frac{\alpha_{0}^{2}}{4 \beta_{0}}\right) .
$$

Similarly, the energy of the single-vortex state is given by

$$
F(L=1)=-\frac{H_{c}(T)^{2}}{\mu_{0}} \xi(T)^{2} d\left(\frac{\alpha_{1}^{2}}{4 \beta_{1}}\right) .
$$

Now, in order to find a height of the potential barrier for vortex entry, we start from the vortex-free solution, $c_{1}=0$. We then begin to increase $c_{1}$ and we minimize $F$ with respect to $c_{0}$ at fixed $c_{1}$. As a result, the following "optimal" dependence of $F$ on $c_{1}$ is obtained

$$
\begin{aligned}
& F(0 \rightarrow 1)=\frac{H_{c}(T)^{2}}{\mu_{0}} \xi(T)^{2} d\left[-\frac{\alpha_{0}^{2}}{4 \beta_{0}}+c_{1}^{2}\left(\alpha_{1}-\frac{\alpha_{0} \gamma}{\beta_{0}}\right)+c_{1}^{4}\left(\beta_{1}-\frac{\gamma^{2}}{\beta_{0}}\right)\right], \quad c_{1} \leq \sqrt{-\frac{\alpha_{0}}{2 \gamma}} \\
& F(0 \rightarrow 1)=\frac{H_{c}(T)^{2}}{\mu_{0}} \xi(T)^{2} d\left(c_{1}^{2} \alpha_{1}+c_{1}^{4} \beta_{1}\right), \quad \sqrt{-\frac{\alpha_{0}}{2 \gamma}}<c_{1} \leq \sqrt{-\frac{\alpha_{1}}{2 \beta_{1}}}
\end{aligned}
$$

and similarly for the vortex exit, $F(1 \rightarrow 0)$. The height of the potential barrier, which is determined by the saddle point in $\left(c_{0}, c_{1}\right)$ space is given by the minimum of $F(0 \rightarrow 1)$ with respect to $c_{1}$ :

$$
U_{e n}=\frac{H_{c}(T)^{2}}{\mu_{0}} \xi(T)^{2} d \frac{\left(\alpha_{1}-\frac{\alpha_{0} \gamma}{\beta_{0}}\right)^{2}}{4\left(\frac{\gamma^{2}}{\beta_{0}}-\beta_{1}\right)} .
$$

A systematic study shows that the vortex-free state is unstable with respect to the vortex penetration at $H \simeq 0.255$ $\mathrm{T}$; while the single-vortex state becomes unstable with respect to the vortex expulsion at $H \simeq 0.195 \mathrm{~T}$. Thus, we see that the maximum hysteresis (in absence of fluctuations) is $\sim 30 \%$. Note that the width of the hysteresis region expands rapidly with increasing $R / \xi(T)$.

In Fig. 1 we plot the dependence of the barrier height (both for vortex entry and exit), given in units of $k_{B} T$, on the external field $H$. We see that heights of both barriers are $\approx 23 k_{B} T$ in the vicinity of $H=0.235 \mathrm{~T}$, where energies of the two phases become equal. The ratio of barrier height and $k_{B} T$ is of the same order as for high- $T_{c}$ materials. The major reasons for a relatively low height of the potential barrier, as compared to the thermal energy, are (i) very small island thickness and (ii) very small disc radius which results in the substantial suppression of the order parameter in the relevant range of applied fields.

In Fig. 2 we show the calculated surface barrier for the vortex entry, given by Eqs. (16), as a function of $c_{1}$. It is quite remarkable that almost the whole interval of $c_{1}$ from $c_{1}=0$ up to the saddle point corresponds to the vortex core nucleation rather than to the motion of a formed vortex inside the island. Fig. 2 thus demonstrates that, under the experimental conditions of Ref. ${ }^{8}$, a vortex position cannot be an appropriate variable to analyze thermal activation over the surface barrier, due to very small island's lateral dimensions. 


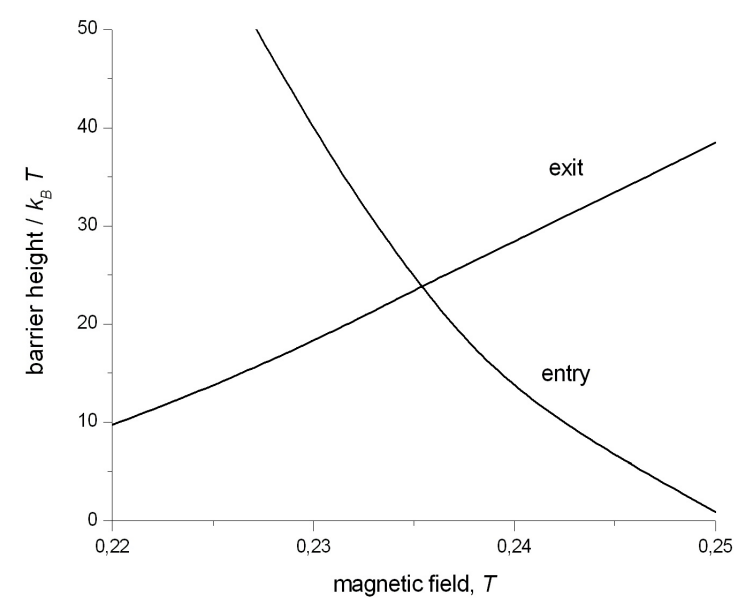

FIG. 1: The dependence of the surface barrier height on the external field $H$.

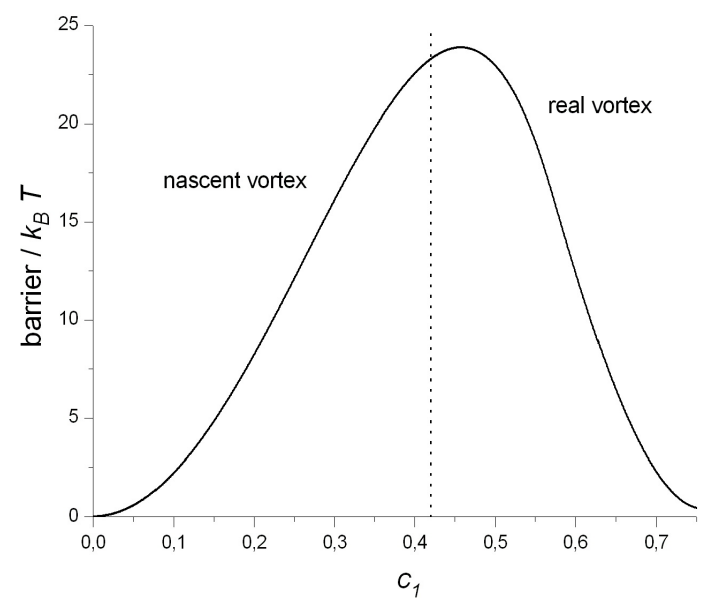

FIG. 2: Potential barrier preventing an increase of $c_{1}$, i.e., a growth of the population of the Landau level with winding number 1 at $H=0.235 \mathrm{~T}$. This growth describes the formation of the vortex nucleus at the island edge with the subsequent motion of a formed vortex towards the island center. Dotted line separates the state with the "nascent" vortex, when the order parameter is still nonzero everywhere, and the phase with the real vortex.

\section{VISCOSITY}

When vortices move, local magnetic field inside a superconductor changes in time. According to the Faraday law, this change produces a nonzero electric field which leads to an energy dissipation. The dissipation can be interpreted in terms of a viscous force acting on moving vortices.

The microscopic theory of this phenomenon is rather complicated. However, reasonable estimates for the viscosity force can be obtained by using phenomenological approach within the Ginzburg-Landau theory 1724 26. In this paper, in order to estimate viscosity associated with the motion of the order parameter, we apply similar ideas.

When finding the island energy, we did not take into account that the magnetic field varies inside the sample. This is fully justified since the disc is very thin $(d \ll \lambda(T))$ and its radius is also small, $R \sim \lambda(T)$, that allows one to use a perturbation theory ${ }^{\sqrt{27}]}$ in terms of a small parameter $1 / \kappa_{\text {eff }}^{2}$, where $\kappa_{\text {eff }}$ is much larger than $\kappa$ due to demagnetization effects. Physically, this implies that the additional field generated by the Meissner current and by the vortex is small 
in comparison with the external uniform field. If we know the solution of the first Ginzburg-Landau equation $f$ for the order parameter in leading order, the vector potential $\mathbf{a}_{a d d}$ of this additional field can be found as

$$
\mathbf{a}_{a d d}=\frac{1}{4 \pi} \int \frac{\mathbf{j}}{r} d V
$$

where an integration is performed over the island volume and $\mathbf{j}$ is a supercurrent density given by

$$
\mathbf{j}=-\frac{1}{\kappa^{2}}\left[\mathbf{a}|f|^{2}+\frac{i}{2}\left(f^{*} \nabla f-f^{*} \nabla f\right)\right]
$$

where $\mathbf{a}=\mathbf{e}_{\varphi} h_{e} r / 2$ is the dimensionless vector potential in leading order which corresponds to the applied uniform field $h_{e}$. Instead of using the exact solution for $f$, we take the lowest Landau level approximation, as done in Section II.

Vortex entry or exit is associated with $f$ changing in time. This change generates the local electric field e, which can be found from the Faraday law

$$
\operatorname{rot} \mathbf{e}=-\frac{\partial\left(\operatorname{rot} \mathbf{a}_{a d d}\right)}{\partial t}
$$

and hence

$$
\mathbf{e}=-\frac{\partial \mathbf{a}_{a d d}}{\partial t}
$$

where $\mathbf{a}_{a d d}$ is given by Eq. (18).

The order parameter inside the island is already strongly suppressed in the vicinity of a transition to the one-vortex state. Therefore, the conductivity is close to the conductivity of $\mathrm{Pb}$ in the normal state $\sigma_{n}$. Then, the dissipation rate in the sample $W$ can be estimated as

$$
W /\left(H_{c 2}(T)^{2} \xi(T)^{5}\right) \sim \sigma_{n} \int \mathbf{e}^{2} d V
$$

where the denominator in the left-hand side comes from the normalization condition.

Let us now consider the process of vortex entry within our representation for the order parameter, as given by Eq. (6). Vortex penetration is associated with the growth in time of $c_{1}$ starting from 0, along the optimal path. It is then easy to see, from Eq. (19), that the leading-order in $c_{1}$ contribution to $\mathbf{j}$ has the following components

$$
\begin{aligned}
& j_{\varphi}=-\frac{1}{\kappa^{2}} c_{0} c_{1} f_{1} f_{0}\left(h_{e} r-\frac{1}{r}\right) \cos \varphi \\
& j_{r}=-\frac{1}{\kappa^{2}} c_{0} c_{1}\left(f_{1 r}^{\prime} f_{0}-f_{0 r}^{\prime} f_{1}\right) \sin \varphi
\end{aligned}
$$

We substitute Eqs. (23) to Eq. (18). Since we are interested in $\mathbf{a}_{\text {add }}$ inside a thin disc, $d \ll R$, we can neglect the dependence on $z$ in the denominator of the integrand in the right-hand side (RHS) of Eq. (18). Then, for the radial component $\mathbf{a}_{a d d}^{(r)}$, after performing integration on angle, we obtain

$$
\mathbf{a}_{a d d}^{(r)}=\frac{d \cos \varphi}{2 \pi \xi(T) \kappa^{2}} c_{0} c_{1} g(r)
$$

where

$$
g(r)=\frac{1}{r} \int_{0}^{r_{0}} r_{1}^{-1}\left(f_{1 r_{1}}^{\prime} f_{0}-f_{0 r_{1}}^{\prime} f_{1}\right)\left[-\left(r_{1}+r\right) E\left(\frac{2 \sqrt{r r_{1}}}{r+r_{1}}\right)+\frac{r^{2}+r_{1}^{2}}{r+r_{1}} K\left(\frac{2 \sqrt{r r_{1}}}{r+r_{1}}\right)\right] d r_{1}
$$

where $K$ and $E$ are complete elliptic integrals of the first and second kind, respectively. Similarly, a $\mathbf{a}_{a d d}^{(\varphi)}$ is found, which is proportional to $\sin \varphi$, while the integrand in the RHS of Eq. (25) now contains $f_{0} f_{1}\left(h_{e} r_{1}-1 / r_{1}\right)$ instead of $\left(f_{1 r_{1}}^{\prime} f_{0}-f_{0 r_{1}}^{\prime} f_{1}\right)$. The motivation to calculate viscosity only in leading order in $c_{1}$ will be clarified in Section IV.

By using Eq. (21), we find the local electric field e as a function of the time derivative $c_{1 t}^{\prime}$

$$
e_{r}=-c_{1 t}^{\prime} \cos \varphi \frac{d}{2 \pi \xi(T) \kappa^{2}} \sqrt{-\frac{\alpha_{0}}{2 \beta_{0}}} g(r),
$$


and similarly for $e_{\varphi}$. The dissipation rate is then determined from Eq. (22) as

$$
W \sim\left(c_{1 t}^{\prime}\right)^{2} \sigma_{n} \delta \frac{H_{c}(T)^{2} \xi(T)^{2} d^{3}}{\kappa^{2}},
$$

where $\delta$ is found from integrating numerically the $r$-dependence of $\mathbf{e}$, as given by Eq. (25), and by using calculated values of $\alpha_{0}$ and $\beta_{0}$. We then arrive to the simple estimate

$$
\delta \sim 10^{-2} .
$$

The "force" of resistance against the growth of $c_{1}$ is given by $W$ divided by $c_{1 t}^{\prime}$. This "force" is proportional to $c_{1 t}^{\prime}$. The viscosity coefficient $\eta^{\left(c_{1}\right)}$, i.e., the proportionality factor, reads

$$
\eta^{\left(c_{1}\right)} \sim \sigma_{n} \frac{H_{c}(T)^{2} \xi(T)^{2} d^{3}}{\kappa^{2}} 10^{-2}
$$

The same expression is found for the viscosity coefficient associated with the growth of $c_{0}$ (the case of a vortex exit). The estimate for $\delta$ remains valid as well.

We stress that $\eta^{\left(c_{1}\right)}$, by definition, is directly linked to the change of the population of the level with winding number 1 and not to the change of the vortex position.

From Eq. (29) we see that $\eta^{\left(c_{1}\right)}$ is proportional to $d^{3}$ and not to $d$, as can be naively expected. Such a strong dependence on $d$ is due to demagnetization effects that significantly diminish the diamagnetic response of the sample and, hence, the electric field. This response is further suppressed due to extremely small lateral dimensions of the island, because the supercurrent created by the vortex is localized within a confined area $\sim \xi(T)^{2}$.

\section{FIRST PASSAGE TIME}

In Sections II and III we have found a profile of the potential barrier and the viscosity in terms of $c_{1}\left(c_{0}\right)$. We now are in a position to find the typical time for the vortex penetration (expulsion). This can be done by using the backward Fokker-Planck equation, also written in terms of $c_{1}\left(c_{0}\right)$.

Let us first consider vortex entrance. The dependence $U\left(c_{1}\right)$ of the system energy on $c_{1}$ is plotted in Fig. 2 . Initially, the system is in the left-hand potential well (vortex-free state, $c_{1}=0$ ). To calculate the average time for the transition to the right-hand potential well (one-vortex state) we use the formalism developed in Ref.28.

We introduce the probability $p\left(c_{1}^{\prime}, t \mid c_{1}, 0\right)$ to find the system at $c_{1}^{\prime}$ in the moment of time $t$, provided it was at $c_{1}$ in the moment $t=0$. The relevant interval of possible values for $c_{1}$ is between the two potential wells, as shown in Fig. $2\left(c_{1} \in[a, b]\right.$, where $\left.a=0, b=\sqrt{-\frac{\alpha_{1}}{2 \beta_{1}}}\right)$. We also denote the saddle-point value of $c_{1}$ as $s$. Then, the probability $G\left(c_{1}, t\right)$ that the system is still in this interval is

$$
G\left(c_{1}, t\right)=\int_{a}^{b} p\left(c_{1}^{\prime}, t \mid c_{1}, 0\right) d c_{1}^{\prime} .
$$

Since the system is homogeneous in time, we can replace $p\left(c_{1}^{\prime}, t \mid c_{1}, 0\right)$ by $p\left(c_{1}^{\prime}, 0 \mid c_{1},-t\right)$, for which the backward Fokker-Planck equation applies

$$
\frac{\partial p\left(c_{1}^{\prime}, 0 \mid c_{1},-t\right)}{\partial(-t)}=\frac{1}{\eta^{\left(c_{1}\right)}} \frac{\partial U}{\partial c_{1}} \frac{\partial p\left(c_{1}^{\prime}, 0 \mid c_{1},-t\right)}{\partial c_{1}}-\frac{k_{B} T}{2 \eta^{\left(c_{1}\right)}} \frac{\partial^{2} p\left(c_{1}^{\prime}, 0 \mid c_{1},-t\right)}{\partial c_{1}^{2}} .
$$

We can now switch back to $p\left(c_{1}^{\prime}, t \mid c_{1}, 0\right)$ and to integrate both sides of Eq. (31) on $c_{1} \in[a, b]$. Taking into account Eq. (30), we obtain an equation for $G\left(c_{1}, t\right)$ :

$$
\frac{\partial G\left(c_{1}, t\right)}{\partial t}=-\frac{1}{\eta^{\left(c_{1}\right)}} \frac{\partial U}{\partial c_{1}} \frac{\partial G\left(c_{1}, t\right)}{\partial c_{1}}+\frac{k_{B} T}{2 \eta^{\left(c_{1}\right)}} \frac{\partial^{2} G\left(c_{1}, t\right)}{\partial c_{1}^{2}} .
$$

We also impose a condition

$$
p\left(c_{1}^{\prime}, 0 \mid c_{1}, 0\right)=\delta\left(c_{1}-c_{1}^{\prime}\right),
$$

which shows that at $t=0$ the system was in the definite state. From this condition and Eq. (30), we get

$$
G\left(c_{1}, 0\right)=1,
$$


which evidences that the system was inside the interval $c_{1} \in[a, b]$ at $t=0$.

We then assume a reflecting condition at $c_{1}=a$, suggesting that the system never leaves $[a, b]$ interval through this end. For the opposite limit, $c_{1}=b$, we impose an absorbing condition, which means that once the system reaches this point, it never enters again the $[a, b]$ interval. We now calculate the average time $\tau\left(c_{1}\right)$, which corresponds to the time, system spends in the interval, provided it was at some fixed $c_{1}$ in the initial moment. Thus, $\tau(a)$ will give us the first passage time from the well at $c_{1}=a$ (vortex-free state) to the well at $c_{1}=b$ (one-vortex state). The time $\tau\left(c_{1}\right)$ can be expressed from $G\left(c_{1}, t\right)$. We first note that $G\left(c_{1}, t\right)$ gives the probability that the system at the moment $t$ is still within the interval $[a, b]$, while $G\left(c_{1}, t+d t\right)$ is the probability that it is still there at $t+d t$. The difference, $-\frac{\partial G\left(c_{1}, t\right)}{\partial t} d t$, represents the probability for the exit within the time interval $[t, t+d t]$. Then, the average exit time $\tau\left(c_{1}\right)$ is given by

$$
\tau\left(c_{1}\right)=-\int_{0}^{\infty} d t\left\{\frac{\partial G\left(c_{1}, t\right)}{\partial t}\right\}=-\int_{0}^{\infty} d G\left(c_{1}, t\right) .
$$

Using Eq. (32) for $G\left(c_{1}, t\right)$, we can obtain the differential equation for $\tau\left(c_{1}\right)$ by integrating both sides of Eq. (32) on $t$ from 0 to $\infty$ :

$$
-\frac{1}{\eta^{\left(c_{1}\right)}} \frac{\partial U}{\partial c_{1}} \frac{\partial \tau\left(c_{1}\right)}{\partial c_{1}}+\frac{k_{B} T}{2 \eta^{\left(c_{1}\right)}} \frac{\partial^{2} \tau\left(c_{1}\right)}{\partial c_{1}^{2}}=-1,
$$

where we have taken into account Eq. (34). Eq. (36) defines a first passage time from arbitrary $c_{1} \in[a, b]$ to $b$. This equation must be supplemented by the conditions for the reflecting boundary at $a$ and absorbing boundary at $b$

$$
\begin{aligned}
\left.\frac{\partial \tau\left(c_{1}\right)}{\partial c_{1}}\right|_{c_{1}=a} & =0, \\
\tau(b) & =0 .
\end{aligned}
$$

The reflecting condition can be obtained from the vanishing of the probability current, while absorbing condition means that the system immediately exits the interval, once it is initially put at the absorbing edge.

Ordinary differential equation (36) with boundary conditions (37) and (38) can be solved by standard methods. Finally, for $\tau(a)$, which will be below denoted as $\tau$, we obtain

$$
\tau \equiv \tau(a)=\frac{2}{k_{B} T} \int_{a}^{b}\left\{\exp \left(\frac{U(y)}{k_{B} T}\right) \int_{a}^{y} \eta^{\left(c_{1}\right)} \exp \left(\frac{-U(x)}{k_{B} T}\right) d x\right\} d y
$$

When integrating over $y$, the dominant contribution to the RHS of Eq. (39) is given by those values of $y$ for which $\exp \left(U(y) / k_{B} T\right)$ is largest, i.e., by the neighborhood of the saddle point $s$ (see Fig. 2). At the same time, the internal integral is essentially constant in this area. It is therefore can be approximated by replacing $y$ in the upper limit of integration by $b$. The RHS of Eq. (39) is then rewritten through a product of two independent integrals

$$
\tau \simeq \frac{2}{k_{B} T}\left(\int_{a}^{b} \exp \left(\frac{U(y)}{k_{B} T}\right) d y\right)\left(\int_{a}^{b} \eta^{\left(c_{1}\right)} \exp \left(\frac{-U(x)}{k_{B} T}\right) d x\right) .
$$

The very high accuracy of such a transformation can be easily verified numerically. It is directly linked to the fact that the barrier height is large compared to $k_{B} T$.

The dominant contribution to the first integral in the RHS of Eq. (40) is provided by the neighborhood of $s$, while for the second integral it is given by the neighborhood of $a$. The latter is basically the reason why, in Section III, we have calculated the viscosity coefficient $\eta^{\left(c_{1}\right)}$ only in leading order in $c_{1}$ : taking into account higher-order terms does not lead to any significant changes. We now expand $U$ in the vicinity of $s$ and $a$ as

$$
\begin{aligned}
& U(x \approx s) \approx U(s)-\frac{\left|U^{\prime \prime}(s)\right|}{2}(x-s)^{2}, \\
& U(x \approx a) \approx U(a)+\frac{\left|U^{\prime \prime}(a)\right|}{2}(x-a)^{2} .
\end{aligned}
$$


It is easy to see that for the simple potential, given by Eq. (16), $\left|U^{\prime \prime}(s)\right|=2\left|U^{\prime \prime}(a)\right|$. After substituting Eqs. (41) and (42) to Eq. (40) and integrating, we obtain

$$
\tau=\tau_{0} \exp \left(\frac{U_{e n}}{k_{B} T}\right)
$$

where $U_{e n}=U(s)-U(a)$ is the barrier height, as given by Eq. (17). The "attempt time" $\tau_{0}$ is

$$
\tau_{0} \approx \frac{\pi \sqrt{2} \eta^{\left(c_{1}\right)}}{U^{\prime \prime}(a)} .
$$

By using Eq. (16), for $U^{\prime \prime}(a)$ we get

$$
U^{\prime \prime}(a)=\frac{2 H_{c}(T)^{2}}{\mu_{0}} \xi(T)^{2} d\left(\alpha_{1}-\frac{\alpha_{0} \gamma}{\beta_{0}}\right)
$$

with $\left(\alpha_{1}-\alpha_{0} \gamma / \beta_{0}\right) \sim 0.1$.

Thus, Eqs. (44) and (45) together with Eq. (29) for $\eta^{\left(c_{1}\right)}$ yield the following estimate for $\tau_{0}$

$$
\tau_{0} \sim \sigma_{n} \mu_{0} \frac{d^{2}}{\kappa^{2}}
$$

We may assume that the conductivity is linear in $T$, so from the known $\sigma_{n}$ of $\mathrm{Pb}$ at room temperature we can estimate $\sigma_{n}$ at the conditions of the experiment $\mathrm{t}^{8}$ as $\sigma_{n} \gtrsim 10^{8} \Omega^{-1} m^{-1}$. Then, $\tau_{0}$ is $\sim 10^{-15}-10^{-14} \mathrm{~s}$. This value together with our result for the surface barrier height, $U_{\text {en }} \approx 23 k_{B} T$, gives $\tau \sim 10^{-5}-10^{-4} \mathrm{~s}$. The very small value for $\tau_{0}$ is due to the fact that the supercurrent is circulating within the tiny volume $\sim \xi(T)^{2} d$, so that the magnetic response of the island is quite weak. Moreover, the order parameter is significantly suppressed. In addition, the distance from the island's edge to its center, which vortex has to pass, is very short.

The above derivation was presented for the case of a vortex entry. The evaluation of the typical time for the vortex exit is quite similar. We have found that Eq. (46) remains applicable, which leads to similar order-of-magnitude estimates for $\tau$.

Experimentally, it has also been observed that the magnetic field corresponding to the transition between one-vortex and vortex-free states was reversible within few percents ${ }^{8}$. This can be understood by noting that, when changing the magnetic field away from $0.235 \mathrm{~T}$, barriers for exit and entrance become asymmetrical. This means that the equilibrium probability distribution becomes extremely strongly peaked within the deeper well, as follows directly from Fig. 2. Indeed, it is easy to see that the difference in heights of the barriers gives the difference in energies between the bottoms of the two wells, because $(U(s)-U(a))-(U(s)-U(b))=U(b)-U(a)$. Since the absolute value of the difference in heights far exceeds $k_{B} T$ already in the vicinity of $0.235 \mathrm{~T}$ (as seen from Fig. 2), the probability to find a system in a more shallow well is $\sim \exp \left(-|U(a)-U(b)| / k_{B} T\right)<<1$. Thus, the system gets stabilized in the deeper well.

Note that $\tau_{0}$, as given by Eq. (46), is not explicitly dependent on $T$, in contrast to the similar quantity calculated in Ref.$^{3}$ within the London approximation. Technically, this is due to the fact that the barrier profile, as given by Eq. (16), is proportional to the second power of the relevant variable (with which $\eta$ is associated), i.e., to $c_{1}^{2}$ at $c_{1} \rightarrow 0$, while the barrier profile in Ref ${ }^{[3}$ is proportional to the first power of vortex displacement when this displacement is small. Through the expansion (42), this affects the second integral in the RHS of Eq. (40) and therefore leads to the mentioned difference. Physically, it might be attributed to the fact that vortex penetration processes for the cases of a half-infinite superconductor and the island in the ultimate vortex confinement regime are qualitatively different. Indeed, in the first case this process is associated with the motion of a "rigid" vortex line inside the superconductor, while in the second case it is represented by the formation of a "soft" vortex nucleus at the edge of the disc. In the first case, the barrier is due to the pronounced magnetic interaction with the superconductor's surface. In the second case, it is due to the condensation energy increase and the residual magnetic interactions.

We also wish to notice that the effect of thermal fluctuations of the order parameter on vortex penetration and expulsion in mesoscopic superconductors was previously studied ${ }^{29}$ by Hernandes et al. by the numerical integration of the time-dependent Ginzburg-Landau equations. In these computations no thermal activation of vortices over the surface barrier was observed for parameters corresponding to samples made of low- $T_{c}$ superconductors, while this activation was shown to be possible for those samples, which are made of high- $T_{c}$ materials. This is consistent with previous experiments on $\mathrm{Al}$ discs ${ }^{30}$ and micron-size structures made of high- $T_{c}$ materia ${ }^{31}$. We think that the difference between the results of the present paper and of Ref. ${ }^{29}$ concerning low- $T_{c}$ samples can be attributed to the fact that in calculations of Ref ${ }^{29}$ the main parameters were chosen in such a way as to model experiments ${ }^{30}$, where 
(i) discs of much larger thickness were studied compared to the island of Ref $[8$ (one order of magnitude and more), (ii) the order parameter was not significantly suppressed due to larger discs radii, (iii) sample temperature was lower in one order of magnitude or so compared to that of Ref $\left[T_{c}\right.$ of $\mathrm{Al}$ is at least several times lower than $T_{c}$ of $\left.\mathrm{Pb}\right)$.

In the very recent experiment 16 , strong current-induced fluctuation phenomena were observed in ultrathin $(\approx 6$ $\mathrm{nm}$ ) superconducting nanowires fabricated in a form of a meander and made of NbN. Lateral dimensions of sample's typical segment, in terms of $\xi(T)$, were one order of magnitude larger than that for the island in the ultimate vortex confinement regime. Structures of this kind are used in single-photon detectors, while fluctuations are major source of dark-count events in such devices. Fluctuation phenomena were explained by a thermally-activated vortex entry, as well as by the unbinding of vortex-antivortex pairs. The height of the surface barrier, as calculated in Ref. 16 , was at least one order of magnitude larger (in terms of $k_{B} T$ ) than the one for the superconducting island studied in the present paper. Nevertheless, vortex penetration events were detectable.

Ref ${ }^{32}$ deals with the experimental investigation of superconducting islands of $\mathrm{Pb}$, which were quite similar to the islands studied in Ref. 8 and in the present paper. The thickness of those islands was even smaller than that of Ref. ${ }^{8}$. However, measurements were performed at significantly lower temperature of $2.0 \mathrm{~K}$. Probably, this is why some hysteresis for vortex penetration and expulsion was observed. Indeed, the activation time depends exponentially on the height of the potential barrier this height being much larger than $k_{B} T$. Nevertheless, it was mentioned in Ref ${ }^{[32}$ that the width of the hysteresis region was noticably smaller than the theoretical values obtained from the Ginzburg-Landau theory. The results of the present paper evidence that thermal fluctuations might be responsible for an observed shrinkage of the hysteresis region.

Let us finally mention a very recent experiment ${ }^{33}$ on one-atomic layer $\mathrm{Pb}$ films, where magnetic vortices were directly visualized. Thermal fluctuations must be strongest in such ultimately thin films.

Since thermal fluctuations are more pronounced in low-dimensional systems, an understanding of vortex matter fluctuations in thin and small superconducting structures is of certain importance. It is howevere clear that more intensive experimental, as well as theoretical efforts are needed to reveal systematically those confinement and temperature regimes which favor thermal fluctuations in such nanostructures or, on the contrary, which enable one to safely avoid them.

\section{CONCLUSIONS}

Although it is generally believed that the surface barrier in low- $T_{c}$ superconductors is too high to be suppressed by thermal fluctuations, in this paper we have shown that such a scenario is possible in small and ultrathin superconducting samples studied in modern experiments. In particular, we have demonstrated that an absence of hysteresis for vortex entry and exit in the nanosized island of $\mathrm{Pb}$ observed in the very recent experiment of Ref ${ }^{8}$ (and the shrinkage of hysteresis region reported in the similar experiment of Ref. ${ }^{[2}$ ) can be explained in terms of a thermal activation of a vortex over the surface barrier.

Lateral dimensions of these islands were so small that they could accommodate only one vortex before gradual transition to the normal state. For this reason, London approximation, which was previously applied to describe thermal surmounting of a surface barrier by vortices, is not applicable.

We therefore have developed an alternative theoretical approach based on the lowest Landau level approximation for the Ginzburg-Landau order parameter, which is suitable for such extremely small structures. The surface barrier profile was calculated in terms of populations of the two relevant Landau levels being used throughout the paper as "good" variables instead of the "bad" one (vortex position). The viscosity coefficient, associated with the motion of the projections of the order parameter, was estimated with account of demagnetization effects. Finally, the expression for the typical time of thermally-assisted vortex entry (exit) was obtained from the Fokker-Planck equation, also written in terms of the populations of Landau levels. This expression is of Arrhenius type.

We have found that the barrier height, under the conditions of experiment ${ }^{8}$, is nearly 20 in terms of a thermal energy $k_{B} T$, which is close to the similar quantity for high- $T_{c}$ materials, where thermal suppression of a surface barrier is observable. Although the exponent of this ratio is still very large, the preexponential "attempt time" is quite small, so that the typical time both for the vortex exit and entrance is several orders of magnitude smaller than $1 \mathrm{~s}$. 


\section{ACKNOWLEDGEMENTS}

Useful discussions with D. Roditchev, T. Cren, and A. L. Rakhmanov are acknowledged. This work was supported by RFBR (project no. 09-02-00248) and "Dynasty Foundation".

${ }^{1}$ C. P. Bean and D. J. Livingston, Phys. Rev. Lett. 12, 14 (1964).

2 E. H. Brandt, Rep. Prog. Phys. 58, 1465 (1994).

3 B. V. Petukhov and V. R. Chechetkin, Zh. Eksp. Teor. Fiz. 65, 1653 (1973) [Sov. Phys. JETP 38, 827 (1974)].

${ }^{4}$ V. N. Kopylov, A. E. Koshelev, I. F. Schegolev, and T. G. Togonidze, Physica C 170, 291 (1990).

${ }^{5}$ L. Burlachkov, Phys. Rev. B 47, 8056 (1993).

6 J. A. Lewis, V. M. Vinokur, J. Wagner, and D. Hinks, Phys. Rev. B 52, R3852 (1995).

7 L. Burlachkov, V. B. Geshkenbein, A. E. Koshelev, A. I. Larkin, and V. M. Vinokur, Phys. Rev. B 50, 16770 (1994).

8 T. Cren, D. Fokin, F. Debontridder, V. Dubost, and D. Roditchev, Phys. Rev. Lett. 102, 127005 (2009).

9 P. S. Deo, V. A. Schweigert, and F. M. Peeters, Phys. Rev. B 59, 6039 (1999); M. N. Kunchur and S. J. Poon, ibid. 43, 2916 (1991); A. R. de C. Romaguera, M. M. Doria, and F. M. Peeters, ibid. 75, 184525 (2007)

10 L. Kramer, Phys. Rev. 170, 475 (1968).

11 V. V. Moshchalkov, X. G. Qiu, and V. Bruyndoncx, Phys. Rev. B 55, 11793 (1997).

12 V. A. Schweigert and F. M. Peeters, Phys. Rev. B 57, 13817 (1998).

13 J. J. Palacios, Phys. Rev. B 58, R5948 (1998).

14 D. S. Golubović, W. V. Pogosov, M. Morelle, and V. V. Moshchalkov, Phys. Rev. Lett. 92, 177904 (2004).

15 M. V. Milošević, A. Kanda, S. Hatsumi, F. M. Peeters, and Y. Ootuka, Phys. Rev. Lett. 103, 217003 (2009).

16 H. Bartolf, A. Engel, A. Schilling, K. Il'in, M. Siegel, H.-W. Hubers, and A. Semenov, Phys. Rev. B 81, 024502 (2010).

17 M. Tinkham, Introduction to Superconductivity, Dover Publications, New York (2004).

18 T. Cren and D. Roditchev, unpublished.

19 J. Pearl, Appl. Phys. Lett. 5, 65 (1964).

20 W. V. Pogosov, Phys. Rev. B 65, 224511 (2002).

21 A. Larkin and A. Varlamov, Theory of Fluctuations in Superconductors, Oxford University Press, Oxford (2004).

22 S. V. Yampolskii and F. M. Peeters, Phys. Rev. B 62, 9663 (2000); J. J. Palacios, F. M. Peeters, and B. J. Baelus, ibid. 64, 134514 (2001).

23 B. J. Baelus, F. M. Peeters, and V. A. Schweigert, Phys. Rev. B 63, 144517 (2001).

${ }^{24}$ L. P. Gorkov and N. B. Kopnin, Usp. Fiz. Nauk 116, 413 (1975) [Sov. Phys. Uspekhi 18, 496 (1976)].

${ }^{25}$ V. V. Schmidt, The physics of superconductors. Introduction to fundamentals and applications, edited by P. Muller and A. V. Ustinov, Springer-Verlag, Berlin (1997).

${ }^{26}$ R. G. Mints and A. L. Rakhmanov, Instabilities in Superconductors, Nauka, Moscow (1984).

27 S. J. Chapman, Q. Du, M. D. Gunzburger, and J. S. Peterson, Adv. Math. Sciences Appl. 5, 193 (1995).

28 C. W. Gardiner, Handbook of Stochastic Methods for Physics, Chemistry and the Natural Sciences, Springer-Verlag, Berlin (2004).

29 A. D. Hernandez, B. J. Baelus, D. Dominguez, and F. M. Peeters, Phys. Rev. B 71, 214524 (2005).

30 A. K. Geim, S. V. Dubonos, I. V. Grigorieva, K. S. Novoselov, F. M. Peeters, and V. A. Schweigert, Nature (London) 407, 55 (2000).

31 J. R. Kirtley, C. C. Tsuei, V. G. Kogan, J. R. Clem, H. Raffy, and Z. Z. Li, Phys. Rev. B 68, 214505 (2003).

${ }^{32}$ T. Nishio, T. An, A. Nomura, K. Miyachi, T. Eguchi, H. Sakata, S. Lin, N. Hayashi, N. Nakai, M. Machida, and Y. Hasegawa, Phys. Rev. Lett. 101, 167001 (2008).

33 T. Zhang, P. Cheng, W.-J. Li, Y.-J. Sun, G. Wang, X.-G. Zhu, K. He, L. Wang, X. Ma, X. Chen, Y. Wang, Y. Liu, H.-Q. Lin, J.-F. Jia, and Q.-K. Xue, Nature Phys. 6, 104 (2010). 